\title{
Limited value of the column concept in operative management of posterior column tibial plateau fractures
}

Juriaan van den Berg, MD 1 ; Stefaan Nijs, MD PhD 1,2 ; Harm Hoekstra, MD PhD FEBS ${ }_{1,2}$

${ }_{1}$ University Hospitals Leuven, Department of Trauma Surgery, B-3000 Leuven, Belgium ${ }_{2}$ KU Leuven - University of Leuven, Department of Development and Regeneration, B-3000 Leuven,Belgium

Corresponding Author: Prof. MD Harm Hoekstra, harm.hoekstra@uzleuven.be, Corresponding address: University Hospitals Leuven, Department of Trauma Surgery, Herestraat 49, B-3000 Leuven, Belgium

Conflict of Interest: The authors declare that they have no conflict of interest.

Funding: There is no funding source.

Ethical approval: This study was completed in compliance with national legislation and the guidelines

of the ethics committee of the University Hospitals Leuven.

Informed consent: Informed consent was obtained from all individual participants included in the study.

\section{ABBREVIATIONS}

PCF : posterior column fracture

TCC : three-column concept

ORIF : open reduction and internal fixation

KOOS : knee injury and osteoarthritis outcome score

\section{KEY-WORDS}

Three-column concept, tibial plateau fractures, posterior column, functional outcome 


\section{ABSTRACT}

Introduction: The three-column concept has been proposed as tool in surgical decision making for treating tibial plateau fractures. Recent studies have underscored the negative effect of posterior column tibial plateau fractures on clinical outcome. The purpose of this study was to assess the value of the three column concept and posterior plating in posterior column fractures. We hypothesized that treating patients according to the three-column concept improves functional outcome.

Methods: 111 consecutive tibial plateau fractures, treated between January 2009 and December 2016, with at least a posterior column fracture were included. Relevant demographic and treatment variables were studied. Applied treatment was retrospectively evaluated according to the threecolumn concept. Patient reported outcome was assessed using the Knee injury and Osteoarthritis Outcome Score (KOOS).

Results: Median follow-up was 43.1 months (IQR 29.0-63.3) with a response rate of 80,2\%. Outcomescores were markedly lower compared to the general population. $22.5 \%$ patients were treated according to the three-column concept and $27 \%$ was treated with posterior plating. Predominantly combined fractures of posterior and lateral columns were treated without fixation of the posterior column. Neither treatment according to the three-column concept and/or with posterior plating were found to significantly influence outcome.

Conclusions: The outcome of posterior column fractures was equal, regardless of whether these fractures were treated or not. This indicates that the three-column concept seems insufficient and gives rise to further debate on surgical strategies of posterior column fractures. The implementation of trauma mechanism based fracture morphology in the threecolumn concept might be important in order to consummate the three-column concept as guiding tool.

\section{INTRODUCTION}

The reported incidence of posterior involvement in proximal tibial plateau fractures ranges from $28.8 \%$ to $70.7 \%$ [1-3]. Outcome in these posterior column fractures (PCF) is not widely described in the literature but recent studies suggest that involvement of the posterior surface of the tibial plateau has more impact on outcome than previously appreciated [2-5]. Sagittal malalignment has been shown to have a negative impact on knee kinematics and functional outcome as well $[5,6]$.

As previously shown, the functional outcome of tibial plateau fractures is markedly better if patients are treated according to the three-column concept (TCC) [3,5]. The TCC guides the surgical approach and implant choice for the treatment of multiple column fractures on the basis of the mechanism of injury and fracture pattern [3]. However, in the past PCF were frequently ignored and therefore we could not determine the benefit of separate PCF fixation. Between 2009 and 2014 only approximately $10 \%$ of all PCF were treated according to TCC principles and fixed in our center [5].

Since 2014 we have been treating patients more often according to the principles of the TCC wherein particularly PCF were managed operatively, assuming that this ultimately will benefit the functional outcome of the patient. This allows us now to assess the importance of open reposition and internal fixation (ORIF) of PCF. To this end, all consecutive tibial plateau fractures involving the posterior column were assessed and evaluated with regard to functional outcome. We hypothesized that ORIF of PCF improves patient reported outcome. 


\section{PATIENTS AND METHODS}

\section{Patients}

All adult patients treated operatively for a tibial plateau fracture between January 2009 and December 2016 were assessed and classified according to the revised three-column classification approach (Figure 1), retrospectively [7]. All patients sustaining a PCF were selected and evaluated for eligibility according to the exclusion criteria. A total of 87 patients were excluded (Figure 2). Pre-existing osteoarthritis was not evaluated. Polytraumatized patients were included, except in cases of bilateral fracture. In addition, treatment strategy in all selected patients was evaluated according to updated TCC principles [3]. This study was completed in compliance with national legislation and the guidelines of the ethics committee of the University Hospitals Leuven.

\section{Study variables}

All patient data were retrieved from the University Hospitals Leuven electronic medical file database KWS. A total of 17 demographic and clinical variables were assessed. Cardiovascular risk factors include current cardiovascular diseases (e.g., CVA, MI, peripheral artery disease), diabetes, obesity, smoking, dyslipidemia, hypercholesterolemia, hypertension, alcohol use, and rheumatoid arthritis. Medication associated with impaired wound healing (e.g., corticosteroids, adrenergic beta-agonists, and chemotherapeutic agents) was recorded. External fixation included all fractures treated using an external fixator in a staged surgical protocol. No patients were treated with an external fixation as definite treatment. Complications were categorized as fracture related infection, nonunion and other tibia related complications (i.e., wound related problems, implant related complaints, compartment syndrome, excessive pain, drop foot, quadriceps muscle atrophy, deep vein thrombosis and neuropraxia). In turn, fracture related infection was defined according to the recent consensus definition [8]. Furthermore, nonunion was assessed using follow-up radiographs and defined according to the US Food and Drug Administration guidelines as a not completely healed fracture within 9 months of injury and without progression toward healing over the past three consecutive months. The quality of the fracture reduction was assessed using $\mathrm{CT}$ if available. The reintervention rate was defined as either implant removal, revision or total knee arthroplasty. Revision was defined as any intervention for loss of reduction, hardware failure or intra-articular hardware.

\section{Outcome measures}

Functional outcome and general health status were assessed using the standardized Knee injury and Osteoarthritis Outcome Score (KOOS) [10]. The KOOS questionnaire is validated for the Dutch language and consists of five subscales; symptoms, pain, activities of daily living, function in sport and recreation and knee related quality of life. Each subscale is presented as a normalized score (100 indicating no symptoms, 0 indicating extremely severe symptoms) and no summarized KOOS score can be constructed due to heterogeneity of the subscales [9]. All eligible patients were sent questionnaires and contacted by telephone if no response was obtained after four weeks.

\section{Statistical analysis}

Statistical evaluation of all data was performed using IBM SPSS 25.0 (SPSS Inc. Chicago, IL). Nominal variables were compared using Chi-Square statistics and nonparametric variables using the Mann- Whitney $\mathrm{U}$ test. For correlation testing the Pearson correlation test was used for continuous variables and the Spearman correlation test for nominal variables. A significance level of $<0.05$ was accepted for all tests. A multivariate analysis was conducted on all significant variables using a linear logistic regression analysis with a stepwise approach.

\section{RESULTS}

\section{Descriptives}

Between January 2009 and December 2016, all 312 consecutive patients presenting with a tibial plateau fracture in our institution were reviewed. After exclusion, a cohort of 111 patients was included in our study (Figure 2). The median follow-up in the study was 43.1 
months (IQR 29.0-63.3). A total of 89 patients responded to the questionnaires, leading to a response rate of $80.2 \%$. In contrast to the responding patients, the nonresponders where more likely to be male $(p=0.010)$, young $(p=0.005)$ and smoking $(p=0.005)$. No other significant demographic or clinical differences between responders and nonresponders were found. The patient, fracture and operative characteristics are summarized in Table 1.

\section{Outcome}

Median KOOS scores for the five subscales are 64.3 (IQR 46.4 - 85.7) for 'symptoms', 62.5 (IQR 44.4 -88.9) for 'pain', 66.2 (IQR 51.5 - 89.7) for 'activities of daily life', 25.0 (IQR 5.0 50.0 ) for 'sports and recreation' and 39.6 (IQR 25.0 - 57.8) for 'knee related quality of life'. With $23(20.5 \%)$ patients stating full flexion and/or extension as impossible. With regard to pain perception, 34 (30.6\%) patients reported experiencing pain on a daily basis and 10 (9.0\%) patients were suffering pain continuously. The median KOOS scores are displayed in Figure 3 in comparison to the general population [10].

\section{TCC, posterior plating and outcome}

Treatment of all patients was assessed for compliance according to the TCC principles. A comparison between demographics and fracture characteristics of patients treated according to the three-column concept and those who were not is displayed in Table 2. In total, 25/111 (22.5\%) patients were treated according to the TCC principles with a disproportionate time distribution ( $p=0.002)$ : 7/61 (11.5\%) before and 18/50 (36.0\%) after implementation of the TCC in 2014. No significant relation between TCC (or no TCC) and any of the KOOS subscales was found in our cohort ( symptoms $p=0.218$, pain $p=0.400$, activities of daily life $p=0.960$, sports and recreation $p=0.853$ and knee related quality of life $p=0.587$, respectively). Furthermore, patients treated between 2014 and 2016 showed also no significant differences in outcome if treated according to TCC principles or not ( symptoms $p=0.091$, pain $p=0.370$, activities of daily life $p=0.159$, sports and recreation $p=0.489$ and knee related quality of life $\mathrm{p}=0.113)$.

In contrast, $30 / 111(27.0 \%)$ of all patients were treated with a posterior plate, including 5 patients who were not fully treated according to the TCC principles (Figure 4): 2/6 (33.3\%) patient with a single (posterior) column fracture, 16/52 (30.8\%) patients with 2 column fractures, and $12 / 53$ patients with 3 column fractures. After implementation of the TCC in 2014 the number of patients treated with a posterior plate doubled: $11 / 61(18.0 \%)$ before and $19 / 50(38.0 \%)$ after implementation, respectively. No significant relation between posterior plate (or not) and any of the KOOS subscales was found (symptoms $p=0.452$, pain $p=0.113$, activities of daily life $p=0.934$, sports and recreation $p=0.789$ and knee related quality of life $p=0.456$, respectively). There was also no significant difference is KOOS scales in the patients treated with a posterior plate between 2014 and 2016: symptoms $p=0.888$; pain $p=0.681$; activities of daily life $p=0.778$; sports and recreation $p=0.961$; knee related quality of life $p=0.707)$. Moreover, the quality of fracture reduction was routinely assessed from 2014 onwards and postoperative CT showed articular incongruence in 46\% (23/50) of these patients.

\section{DISCUSSION}

The aim of this study was to investigate the value of the TCC in the treatment of tibial plateau fractures, including posterior column fractures and determine whether or not there is benefit of posterior fixation. We hypothesized that ORIF of PCF might improve patient reported outcome. Therefore, we examined all operatively treated tibial plateau fractures with a PCF between 2009 and 2016 in our center, and assessed whether or not the operative management was according to the TCC principles with adequate fixation of the PCF. Surprisingly, we could not reveal a significant benefit of TCC with regard to the functional outcome. Although the TCC was implemented as early as 2014, only well over a third of the patients were treated according to the TCC and did not show significant differences in KOOS subscales. Apart from whether patients were treated according to the TCC, also patients treated with posterior plating did not show better outcome scores.

The two column fractures mainly concerned combined posterior and lateral column fractures (Table 1), which were treated using single lateral plate fixation predominantly. Using variable angle - locking compression plates (VA-LCP) with a 30 degrees' cone allows us to diverge the 
VA-LCP locking (rafting) screws posteriorly through the fracture and ensure adequate fixation and articular support of extended lateral column fractures (Figure 1). Nevertheless, lateral ORIF does not always provide sufficient support of posterolateral (and posteromedial) column fractures (Figure 1) [11-13]. Surely enough, only $3 / 42$ patients $(7.1 \%)$ with a combined lateral and posterior column fracture were treated according to the TCC (i.e. lateral and posterior ORIF). No statistically significant difference in outcome was noted, although these numbers are insufficient to draw conclusions from.

A frequently observed fracture pattern was a lateral tibial plateau fracture with an anteroposterior fracture configuration, and depression or sagittal malalignment or depression. Strict evaluation of these fractures according to the TCC indicates a combined lateral and posterior column fracture. As shown by Molenaars et al. such lateral split fracture pattern are frequent and can occur in up to $75 \%$ of all tibial plateau fractures [2]. Although optimal treatment strategy for this type of fracture can further be debated (i.e. fracture morphology, degree of articular depression), a lateral split (depressed) fracture should sufficiently be treated via a single lateral approach. On the contrary, if a fracture of the posterior wall is present in a more lateromedial direction (shear fracture), additional posterior fixation with adequate buttressing according to the TCC seems necessary [2-3]. This shows that there is a flaw in the TCC and might also be an explanation for the fact that we have not been able to show any added value of the TCC in patients sustaining a PCF. Fracture morphology is indeed important in the choice whether or not to treat posterior column fractures. The implementation of trauma mechanism based fracture morphology can be an important link in order to consummate the TCC and guide future surgical decision making with regard to the fixation of the posterior column [14]. The 10-segment classification proposed by Krause et al., being more exhaustive towards recognizing articular fracture patterns and associated ligamentous injury, is not likely to offer added value towards prediction of secondary displacement or surgical planning [15]. Therefore, further investigation into current techniques and hardware to assess for efficacy in the treatment of these fractures is needed.

We are aware that the current study has some limitations. First, the retrospective design has to some degree an inherent selection bias. Second, to a certain extent surgical decision making causes an inherent heterogeneity due to treatment selection bias. In other words, a severe fracture with comminution potentially could have a higher probability of receiving posterior fixation. Furthermore, the long inclusion period encompasses different implemented treatment strategies over time. Finally, the cross-sectional design leads to a variable follow-up time of the measured outcome. Nevertheless, this study represents a relatively large cohort of consecutive patients in a single large level 1 trauma center, with a structured management, rehabilitation and follow-up protocol. Furthermore, the relatively high response rate and diverse patient characteristics make this cohort representable for study purposes.

In conclusion, the outcome scores of patients after sustaining a PCF were markedly lower than values in the reference population. Although the current revised three-column classification approach is very helpful toward surgical planning, in particular of extended lateral column fractures [11], there are still multiple issues that need to be addressed. Fracture patterns of multiple column fractures seem to be more comprehensive than can be adequately expressed by the TCC. Fracture morphology and the risk for secondary displacement are case specific and although thoroughly investigated with CT-images, no current classification seems to fully satisfy. Case selection for posterior fixation is still highly debated and our current findings can't sufficiently direct our decision making. Further investigation into fracture morphology in relation to trauma mechanisms and surgical decision making needs to be performed in order to adequately treat the diversity of fracture patterns. 3D-classification is a relatively new concept that could be investigated towards predicting outcome and give direction to surgical planning of these complicated fractures. Furthermore, prospective study protocols with adequate fixation of selected posterior column fractures and extensive review of the outcome are needed.

\section{COMPLIANCE WITH ETHICAL STANDARDS}


Conflict of interest: The authors declare that they have no conflict of interest.

Ethical approval: This study was completed in compliance with national legislation and the guidelines of the ethics committee of the University Hospitals Leuven.

Informed consent: Informed consent was obtained from all individual participants included in the study.

\section{LEGENDS}

\section{Figure 1}

The revised three-column classification (rTCC) according to Hoekstra et al. [7], depicting the posterior column $(\mathrm{OBC})$ which should be treated via a posterior approach. Lateral column $(O A C)$ fractures that extent into the posterolateral corner (dotted area) are defined as extended lateral column fractures $(O A D)$ and can sufficiently be treated via a single lateral approach using VA-LCPs [11].

\section{Figure 2}

Patient selection diagram

\section{Figure 3}

KOOS subscale outcome scores of study cohort, compared to a reference population [13]. Abbreviations: KOOS, Knee injury and Osteoarthritis Outcome Score; ADL, activities of daily living; Sport/Rec, function in sport and recreation; QoL, knee related quality of life.

\section{Figure 4}

53-year-old female who sustained a three-column tibial plateau fracture. A, preoperative CT including respectively coronal, sagittal and axial view. $B$, initial treatment consisted of a single VA-LCP osteosynthesis. C, postoperative CT revealed coronal and sagittal malalignment due to a collapsed lateral tibial plateau and an unstable posterior column fracture (respectively coronal, sagittal and axial view). D, reosteosynthesis with posterior buttress of the posterior column fracture and ORIF of the extended lateral column using a VA-LCP and a K-wire. The combined (antero-) lateral and posteromedial reversed L-shaped approach provide here an extensive exposure of both the joint (whole extended lateral column) as well as the posterior aspect of the proximal tibia. After arthrotomy and tibia condyle osteotomy, the exposure of the joint can be increased by inserting a small retractor behind the posterior wall just medially of the fibular head.

\section{REFERENCES}

1. Molenaars RJ, Mellema JJ, Doornberg JN, Kloen P (2015) Tibial Plateau Fracture Characteristics: Computed Tomography Mapping

of Lateral, Medial, and Bicondylar Fractures. J Bone Joint Surg Am 97:1512-20 . doi: 10.2106/JBJS.N.00866

2 Yang G, Zhai Q, Zhu Y, et al (2013) The incidence of posterior tibial plateau fracture: an investigation of 525 fractures by using a CTbased

classification system. Arch Orthop Trauma Surg 133:929-34 . doi: 10.1007/s00402-013-1735-4

3. Wang Y, Luo C, Zhu Y, et al (2016) Updated Three-Column Concept in surgical treatment for tibial plateau fractures - A prospective

cohort study of 287 patients. Injury 47:1488-1496 . doi: 10.1016/j.injury.2016.04.026

4. Jiwanlal A, Jeray KJ (2016) Outcome of Posterior Tibial Plateau Fixation. J Knee Surg 29:34 -9 . doi: 10.1055/s-0035-

1564729

5. van den Berg J, Reul M, Nunes Cardozo M, et al (2017) Functional outcome of intra-articular tibial plateau fractures: the impact of

posterior column fractures. Int Orthop 41:1865-1873 . doi: 10.1007/s00264-017-3566-3

6. Ahmad R, Patel A, Mandalia V, Toms A (2016) Posterior Tibial Slope: Effect on, and Interaction with, Knee Kinematics. JBJS Rev

4:e3-e3 . doi: 10.2106/JBJS.RVW.0.00057

7 Hoekstra H, Kempenaers K, Nijs S (2017) A revised 3-column classification approach for the surgical planning of extended lateral

tibial plateau fractures. Eur J Trauma Emerg Surg 43:637-643 . doi: 10.1007/s00068-016-0696-z

8. Metsemakers WJ, Morgenstern M, McNally MA, et al (2017) Fracture-related infection: A consensus on definition from an international expert group. Injury 6-11 . doi: 10.1016/j.injury.2017.08.040

9. Roos EM, Lohmander LS (2003) The Knee injury and Osteoarthritis Outcome Score (KOOS): from joint injury to osteoarthritis. Health

Qual Life Outcomes 1:64 . doi: 10.1186/1477-7525-1-64

10. de Groot IB, Favejee MM, Reijman M, et al (2008) The Dutch version of the Knee Injury and Osteoarthritis Outcome

Score: a

validation study. Health Qual Life Outcomes 6:16 . doi: 10.1186/1477-7525-6-16

11. Hoekstra, H., Vanhees, J., van den Berg, J., \& Nijs, S. (2018). Extended lateral column tibial plateau fractures. How do we do it?

Injury. http://doi.org/10.1016/j.injury.2018.07.027

12. Chang SM, Hu SJ, Du SC, et al (2018) Four-quadrant/column classification of tibial plateau fractures. Int Orthop 42:725727 . doi:

10.1007/s00264-017-3733-6

13. Orapiriyakul W, Apivatthakakul T, Phornphutkul C (2018) Posterolateral tibial plateau fractures, how to buttress?

Reversed L 
posteromedial or the posterolateral approach: a comparative cadaveric study. Arch Orthop Trauma Surg 138:505-513 . doi: 10.1007/s00402-018-2875-3

14. Millar SC, Arnold JB, Thewlis D, et al (2018) A systematic literature review of tibial plateau fractures: What classifications are used

and how reliable and useful are they? Injury 49:473-490 . doi: 10.1016/j.injury.2018.01.025

15. Krause M, Preiss A, Muller G, et al (2016) Intra-articular tibial plateau fracture characteristics according to the "Ten

segment

classification." Injury 47:2551-2557 . doi: 10.1016/j.injury.2016.09.014 
Figure 1

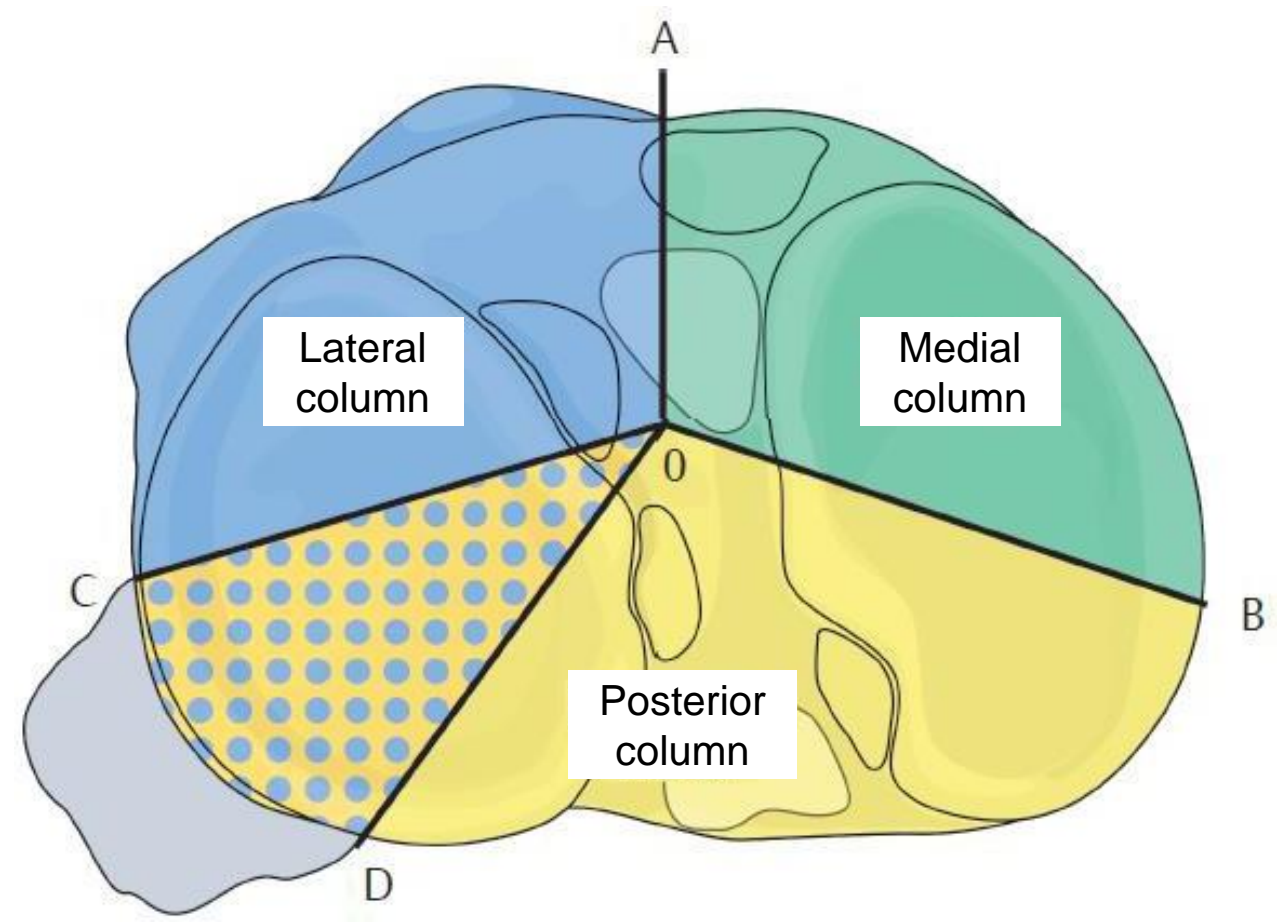

Figure 1 
Figure 2

312 consecutive operatively treated patients with proximal tibial plateau fractures

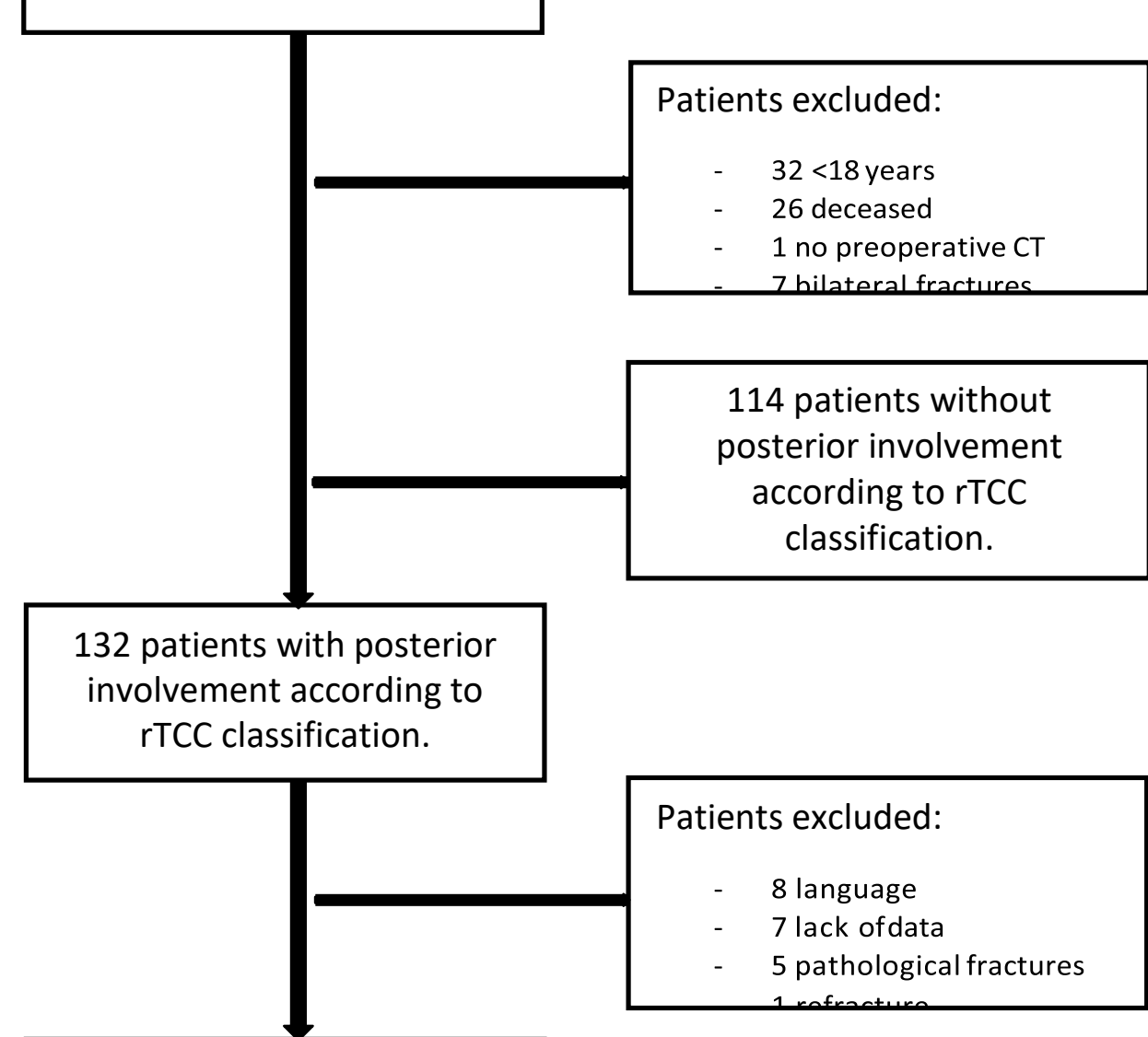

111 posterior tibial plateau fractures included 
Figure 3

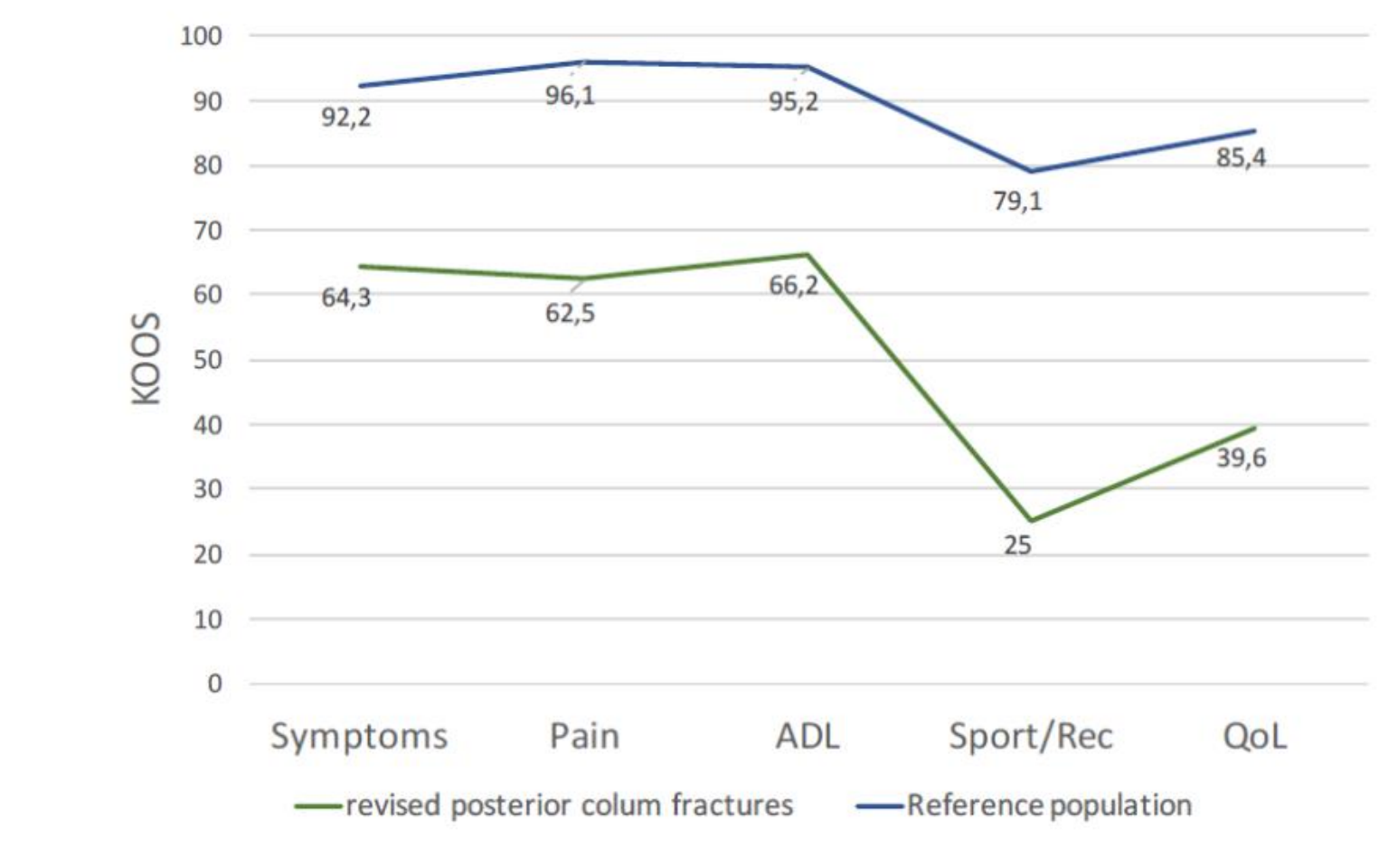

revised posterior colum fractures _ Reference population

\section{Figure 3 s.}

Figure 3

3

政
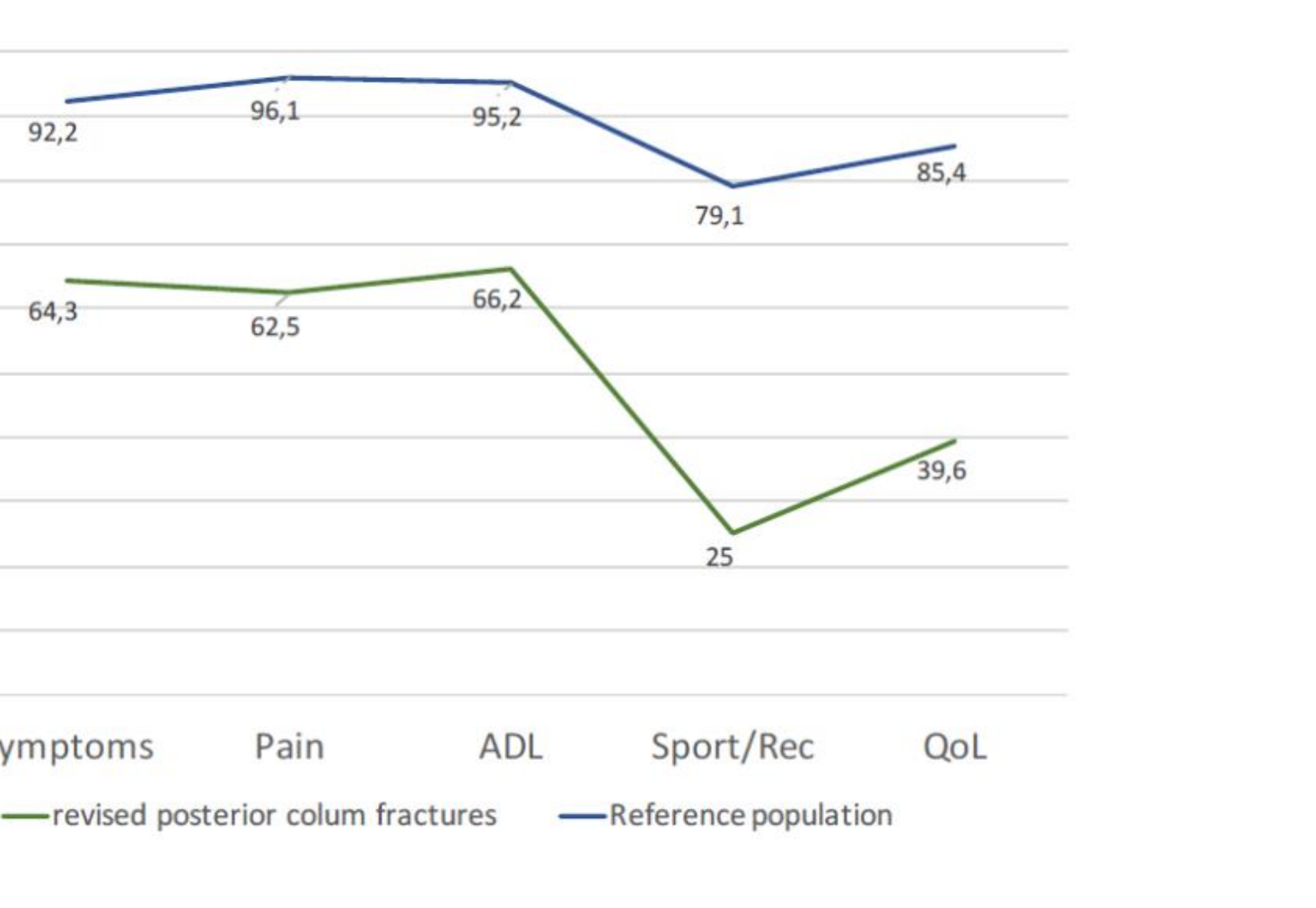


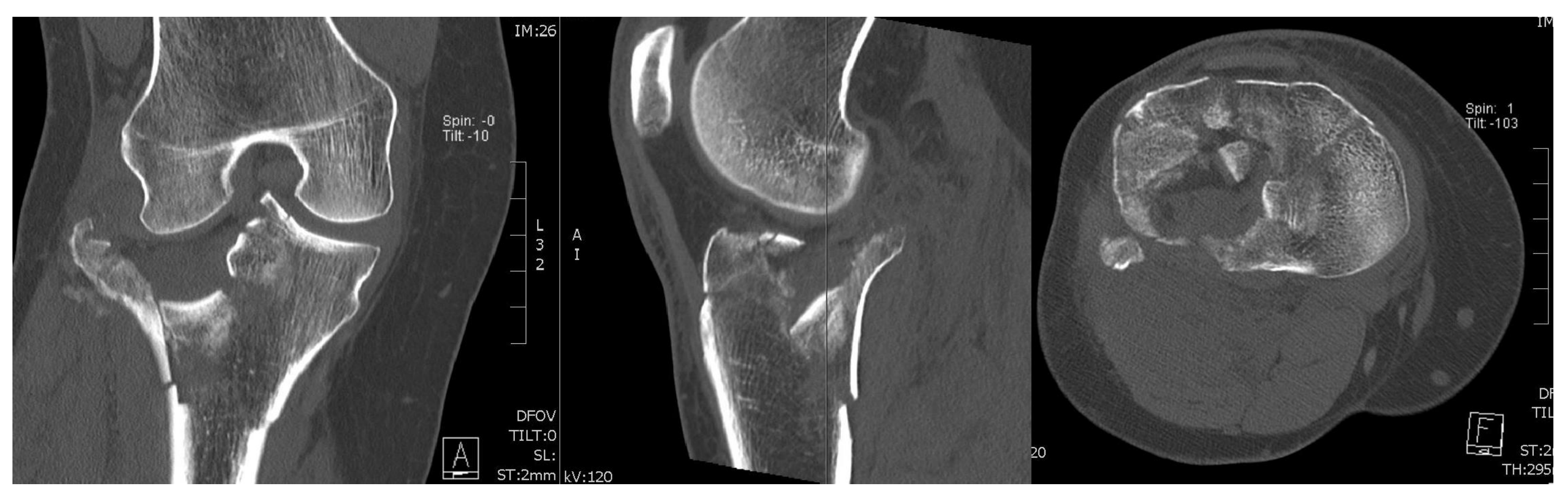

Figure 4A 


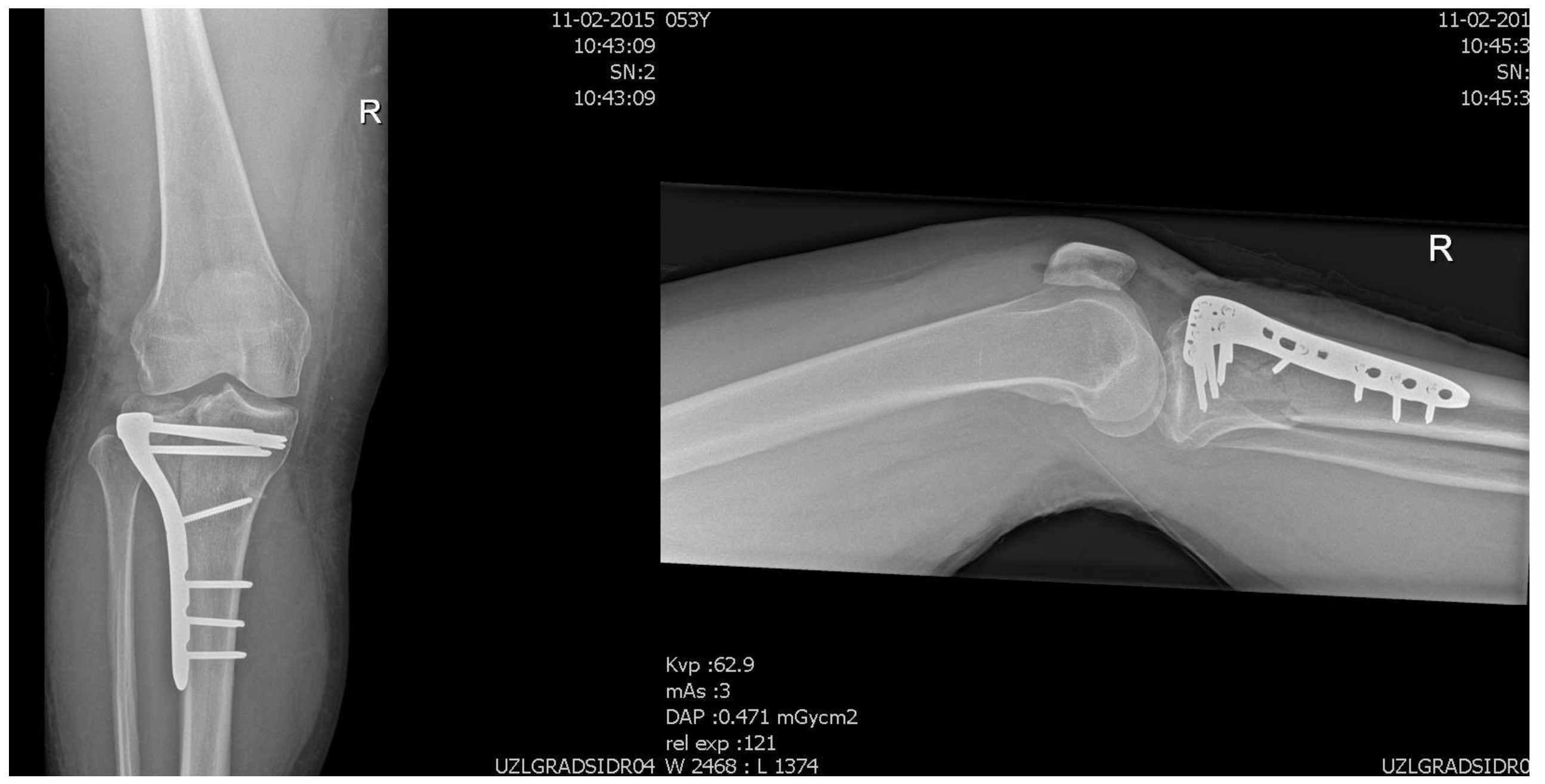

Figure 4B 

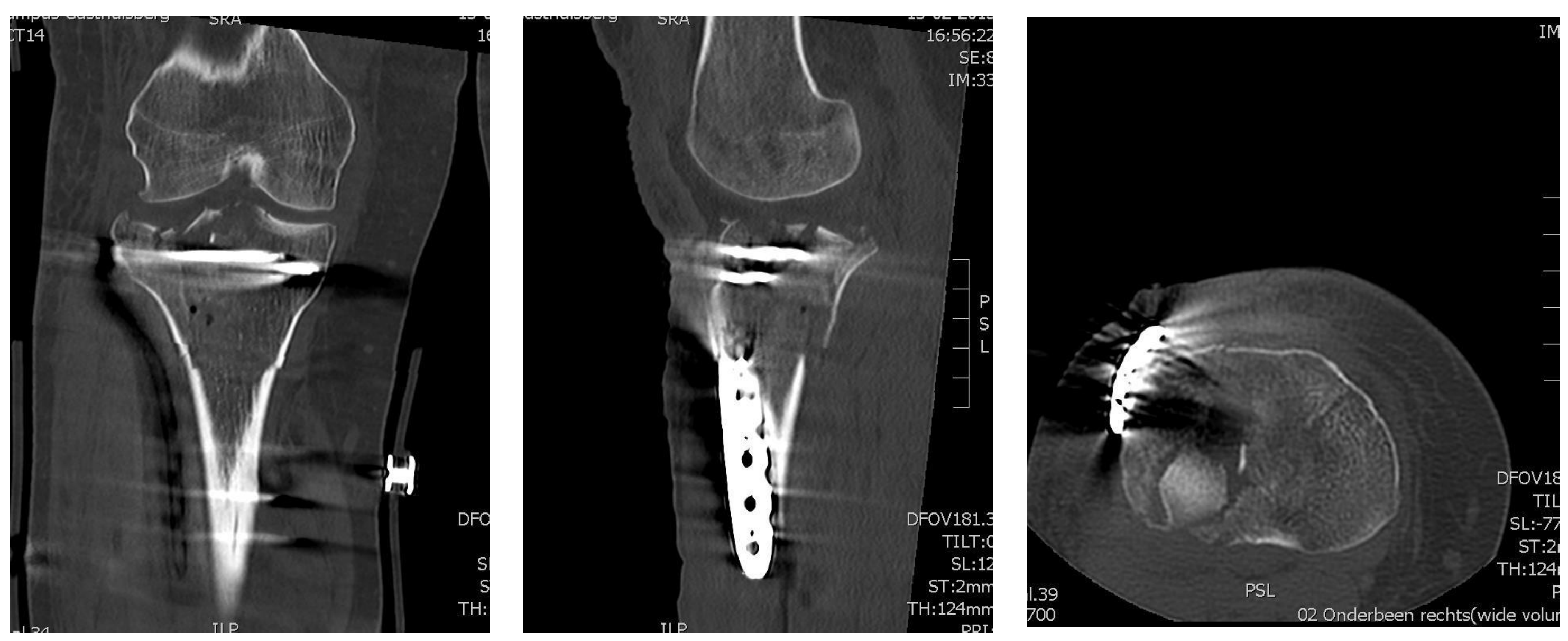

Figure 4C 


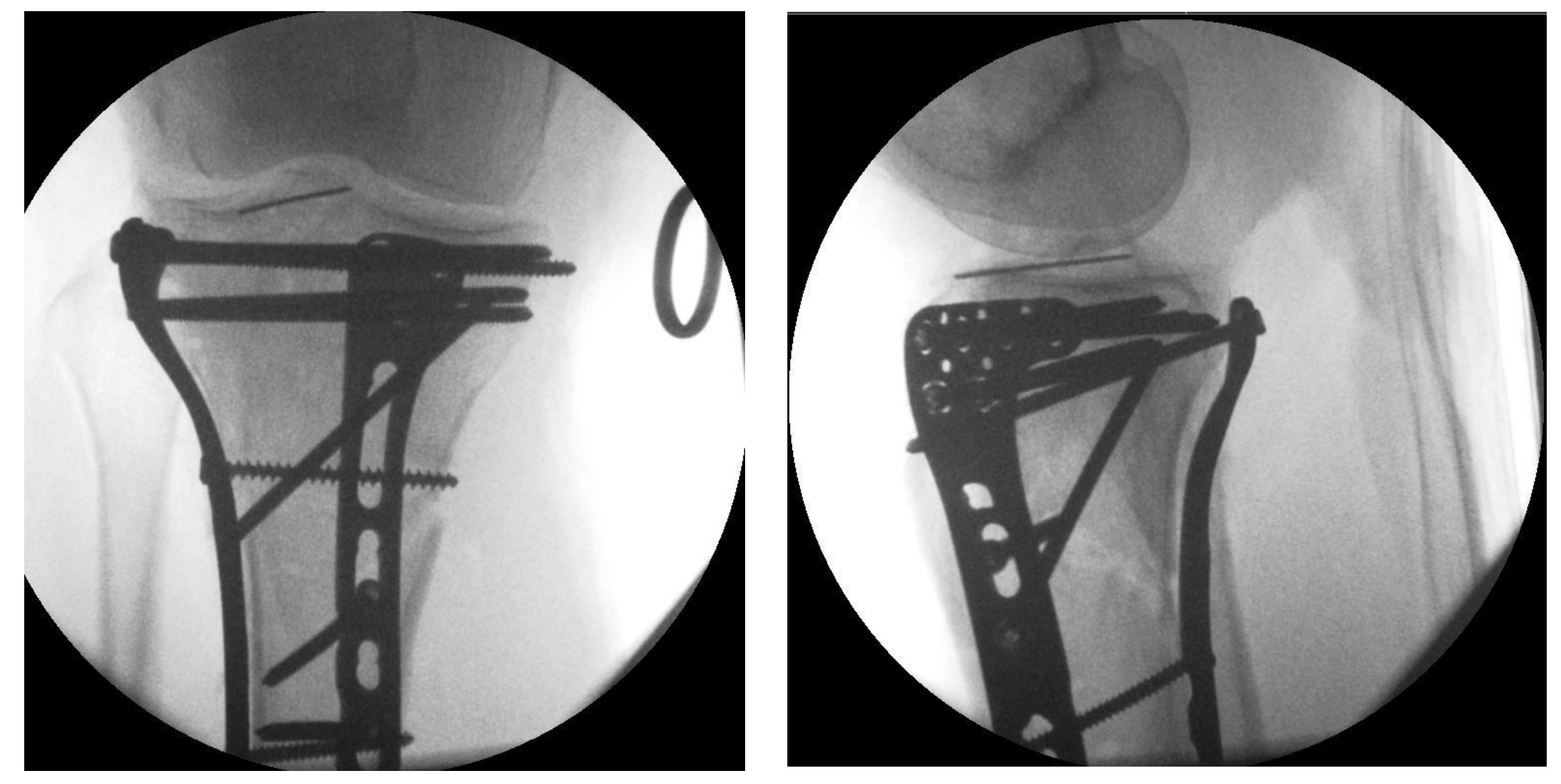

Figure 4D 


\begin{tabular}{|c|c|}
\hline Age (years) & $\begin{array}{l}52.6,51.6 \\
(42.7-61.1,15.3, \\
21.2-91.0)\end{array}$ \\
\hline \multicolumn{2}{|l|}{ Gender } \\
\hline Male & $44(39.6 \%)$ \\
\hline Female & $67(60.4 \%)$ \\
\hline \multicolumn{2}{|l|}{ ASA-score } \\
\hline 1 & $36(32.4 \%)$ \\
\hline 2 & $65(58.6 \%)$ \\
\hline 3 & $9(8.1 \%)$ \\
\hline 4 & $1(0.9 \%)$ \\
\hline \multicolumn{2}{|l|}{ CVRF } \\
\hline $\mathrm{BMI}\left(\mathrm{kg} / \mathrm{m}^{2}\right)$ & $\begin{array}{l}26.3,26.8(22.9- \\
29.3,4.9,18.3-\end{array}$ \\
\hline Smoking & 42.1) \\
\hline Medication & $29(26.1 \%)$ \\
\hline DM & $27(24.3 \%)$ \\
\hline \multirow[t]{2}{*}{ Other CVRF } & $9(8.1 \%)$ \\
\hline & 44 (39.6\%) \\
\hline Side & $71(64.0 \%)$ \\
\hline Left & $40(36.0 \%)$ \\
\hline Right & $8(7.2 \%)$ \\
\hline Open fracture & $8(7.2 \%)$ \\
\hline \multicolumn{2}{|l|}{ rTCC classification } \\
\hline 1 column & $6(5.8 \%)$ \\
\hline 2 columns & $52(46.8 \%)$ \\
\hline posterior + medial & 10 \\
\hline posterior + lateral & 42 \\
\hline 3 columns & $53(47.7 \%)$ \\
\hline External fixation & $18(16.2 \%)$ \\
\hline \multicolumn{2}{|l|}{ Delayed (-staged) surgery } \\
\hline Direct $(<24 \mathrm{hrs})$ & $17(15.3 \%)$ \\
\hline Delayed (>24 hrs) & $94(84.7 \%)$ \\
\hline Time to surgery (days) & $\begin{array}{l}4,5.15(2-7,4.6 \\
0-24)\end{array}$ \\
\hline Complication rate & $43(38.7 \%)$ \\
\hline Fracture related infection & $13(11.7 \%)$ \\
\hline Nonunion & $5(4.5 \%)$ \\
\hline Other complications & $34(30.6 \%)$ \\
\hline Reintervention rate & $49(44.1 \%)$ \\
\hline Implant removal & $40(36.0 \%)$ \\
\hline Revision & $17(15.3 \%)$ \\
\hline TKA & $8(7.2 \%)$ \\
\hline
\end{tabular}

Continuous parameters are expressed as median and mean values with their respective interquartile range, standard deviation and range. Categorical variables are expressed as numbers and percentages of the total number of included patients ( $n=111$ ). Abbreviations: ASA, American Society of Anesthesiologists; CVRF, cardiovascular risk; BMI, body mass index; DM, diabetes mellitus; rTCC, revised three-column classification; TKA, total knee arthroplasty. 
Table 2

Table 2. Treatment according to three-column concept and not $(n=111)$

\begin{tabular}{|c|c|c|c|}
\hline & $\operatorname{TCC}(n=25)$ & no TCC $(n=86)$ & P-value \\
\hline Age (years) & $\begin{array}{l}53.9,52.8(42.5- \\
62.9,14.7,24.0- \\
81.4)\end{array}$ & $\begin{array}{l}51.7,51.3(42.3-60.7 \\
15.5,21.2-91.0)\end{array}$ & 0.525 \\
\hline Gender & & & 0.984 \\
\hline Male & $10(22.7 \%)$ & $34(77.3 \%)$ & \\
\hline Female & $15(22.4 \%)$ & $52(77.6 \%)$ & \\
\hline ASA-score & & & 0.944 \\
\hline 1 & $9(25.0 \%)$ & 27 (75.0\%) & \\
\hline 2 & $13(20.0 \%)$ & $52(80.0 \%)$ & \\
\hline 3 & $3(33.3 \%)$ & $6(66.7 \%)$ & \\
\hline 4 & 0 & $1(100 \%)$ & \\
\hline \multicolumn{4}{|l|}{ CVRF } \\
\hline BMI $\left(\mathrm{kg} / \mathrm{m}^{2}\right)$ & $\begin{array}{l}27.4,28.5(25.2- \\
32.2,4.9,18.8- \\
40.4)\end{array}$ & $\begin{array}{l}25.7,26.3(22.6-29.0 \\
4.7,18.3-42.1)\end{array}$ & $0.031 *$ \\
\hline Smoking & $5(17.2 \%)$ & $24(82.8 \%)$ & 0.445 \\
\hline Medication & $7(25.9 \%)$ & $20(74.1 \%)$ & 0.717 \\
\hline DM & $4(44.4 \%)$ & $5(55.6 \%)$ & 0.097 \\
\hline Other CVRF & $10(22.7 \%)$ & $34(77.3 \%)$ & 0.950 \\
\hline Side & & & 0.612 \\
\hline Left & $15(21.1 \%)$ & $56(78.9 \%)$ & \\
\hline Right & $10(25.0 \%)$ & $30(75.0 \%)$ & \\
\hline Open fracture & $1(12.5 \%)$ & $7(87.5 \%)$ & 0.481 \\
\hline rTCC classification & & & $0.038 *$ \\
\hline 1 column (posterior) & $2(33.3 \%)$ & $4(66.7 \%)$ & \\
\hline 2 columns & $5(9.6 \%)$ & $47(90.4 \%)$ & \\
\hline posterior + medial & $2(20.0 \%)$ & $8(80.0 \%)$ & \\
\hline posterior + lateral & $3(7.1 \%)$ & 39 (92.9\%) & \\
\hline 3 columns & $18(34.0 \%)$ & 35 (66.0\%) & \\
\hline
\end{tabular}

Comparison between demographics and fracture characteristics of patients treated according to the threecolumn concept and those who were not. Continuous parameters are expressed as median and mean values with their respective interquartile range, standard deviation and range. Percentage displayed is according to the respective treatment category. The respective P-values for all variables are calculated between treatment groups using Chi- Square testing for binominal, ANOVA for multinominal and MannWhitney $\mathrm{U}$ test for continuous variables.

Abbreviations:TCC, three-column concept; ASA, American Society of Anesthesiologists; CVRF, CardiovascularRisk Factors; BMI, Body Mass Index; DM, Diabetes Mellitus; rTCC, revised threecolumn classification. 